\title{
EXTREMAL QUASICONFORMAL MAPPINGS WITH PRESCRIBED BOUNDARY VALUES
}

\author{
BY \\ RICHARD S. HAMILTON
}

1. Let $R$ be a Riemann surface whose universal covering space is conformally equivalent to the unit disk. We can regard $R$ as the interior of a Riemann surface with boundary $R^{*}$ whose boundary is as large as possible (see $\S 3$ ). A quasiconformal map $f$ of $R$ onto another Riemann surface $S$ has a unique continuous extension $f^{*}$ mapping $R^{*}$ onto $S^{*}$. Two quasiconformal maps $f$ and $g$ of $R$ onto $S$ are homotopic modulo the boundary if $f^{*}=g^{*}$ on $R^{*}-R$ and there exists a homotopy between $f^{*}$ and $g^{*}$ which is constant on $R^{*}-R$. If $R^{*}-R$ is empty then $f$ is just homotopic to $g$.

Let $K$ be the complex cotangent bundle of $R$. Then $\beta(f)$, the Beltrami differential of $f$, is an element of $L^{\infty}\left(\bar{K} K^{-1}\right)$, the Banach space of all essentially bounded sections of the bundle $\bar{K} K^{-1}$. (Locally $\nu \in L^{\infty}\left(\bar{K} K^{-1}\right)$ is given by $v_{\alpha} d \bar{z}_{\alpha} / d z_{\alpha}$.) $L^{\infty}\left(\bar{K} K^{-1}\right)$ is the dual of $L^{1}\left(K^{2}\right)$, the Banach space of integrable sections of the bundle $K^{2}$. (Locally $\varphi \in L^{1}\left(K^{2}\right)$ is given by $\varphi_{\alpha} d z_{\alpha}^{2}$.) Let $A(R)$ denote the closed subspace of $L^{1}\left(K^{2}\right)$ of all integrable analytic quadratic differentials on $R$. By $\|\beta(f) \mid A(R)\|$ we denote the norm of the restriction of $\beta(f)$, regarded as a linear functional on $L^{1}\left(K^{2}\right)$, to the closed subspace $A(R)$.

The quasiconformal map $f: R \rightarrow S$ is extremal if $\|\beta(f)\| \leqq\|\beta(g)\|$ for all maps $g$ homotopic to $f$ modulo the boundary. The main result is

THEOREM 1. If $f: R \rightarrow S$ is extremal then $\|\beta(f) \mid A(R)\|=\|\beta(f)\|$.

COROllaRY 1. If $R$ is a compact surface minus a finite number of points, then $A(R)$ is finite dimensional and consists of all meromorphic quadratic differentials on the compact surface with poles of order at most one at the deleted points. Furthermore there exists a nonzero $\theta \in A(R)$ and a constant $c$ with $\beta(f)=c \tilde{\theta} /|\theta|$.

REMARK. This result is classical and due to Teichmüller.

Proof. Since $A(R)$ is finite dimensional, by Theorem 1 there exists a nonzero $\theta \in A(R)$ with $\int_{R} \beta(f) \theta=\int_{R}\|\beta(f)\||\theta|$. This can happen only if $\beta(f) \theta=\|\beta(f)\||\theta|$, and therefore $\beta(f)=\|\beta(f)\| \tilde{\theta} /|\theta|$.

COROllaRY 2. If $f: R \rightarrow S$ is extremal then $\|\beta(f) \mid R-K\|=\|\beta(f)\|$ for every compact proper subset $K$ of $R$.

Proof. By Theorem 1 we can find a sequence $\theta_{n} \in A(R)$ with $\left\|\theta_{n}\right\|=1$ and $\int_{R} \beta(f) \theta_{n} \rightarrow\|\beta(f)\|$. Since the value of an analytic function at a point is the average

Received by the editors June 9, 1967 and, in revised form, March 3, 1968. 
of its values over a disk centered at that point, the $\theta_{n}$ are uniformly bounded on every compact subset. Using Cauchy's formula their derivatives are uniformly bounded also, and by passing to a subsequence we may assume that the $\theta_{n}$ converge uniformly on compact subsets to some $\theta \in A(R)$. Suppose $\|\beta(f) \mid R-K\|<\|\beta(f)\|$. Since

$$
\left|\int_{R} \beta(f) \theta_{n}\right| \leqq\|\beta(f)\| \int_{K}\left|\theta_{n}\right|+\left\|\beta(f)\left|R-K \| \int_{R-K}\right| \theta_{n} \mid\right.
$$

we must have $\int_{R-K}\left|\theta_{n}\right| \rightarrow 0$, and the $\theta_{n}$ converge to $\theta$ in $L^{1}$ norm. We then have $\|\theta\|=1$ and $\beta(f)(\theta)=\|\beta(f)\|$, and we can repeat the previous argument to show $\beta(f)=\|\beta(f)\| \bar{\theta} /|\theta|$. This gives a contradiction.

REMARK. Given any quasiconformal map $f: R \rightarrow S$, it follows from the usual compactness properties of quasiconformal mappings that there exists at least one extremal map homotopic to $f$ modulo the boundary. Strebel [7] has shown that when $R$ is the unit disk such an extremal need not have the form $\beta(f)=c \bar{\theta} /|\theta|$ and need not be unique.

2. The proof of Theorem 1 will be modeled upon the following general result.

THEOREM 2. Let $B$ be a Banach space and $B^{*}$ its dual. Let $M$ be a $C^{1}$ submanifold of $B^{*}$. Suppose that the dual norm in $B^{*}$ assumes its minimum, or maximum, on $M$ at a point $x$ in $M$, and that there exists a closed subspace $A$ of $B$ such that the tangent space to $M$ at $x$ is the subspace of $B^{*}$ orthogonal to $A$. Then $\|x \mid A\|=\|x\|$.

Proof. Suppose $\|x \mid A\|<\|x\|$. By the Hahn-Banach extension theorem we can find a linear functional $y$ in $B^{*}$ with $y|A=x| A$ and $\|y\|=\|x \mid A\|<\|x\|$. Since $y-x$ vanishes on $A$, we can construct a $C^{1}$ path $\alpha:(-\varepsilon, \varepsilon) \rightarrow M$ with $\alpha(0)=x$ and $D \alpha(0)(1)=y-x$.

Then for sufficiently small positive $t$

$$
\|\alpha(t)-\alpha(0)-D \alpha(0)(t)\| / t<\|x\|-\|y\|
$$

since $\|x\|-\|y\|>0$. But then

$$
\begin{aligned}
\|\alpha(t)\| & <\|\alpha(0)+D \alpha(0)(t)\|+(\|x\|-\|y\|) t \\
& \leqq\|x+t(y-x)\|+(\|x\|-\|y\|) t \\
& \leqq(1-t)\|x\|+t\|y\|+t\|x\|-t\|y\|=\|x\|
\end{aligned}
$$

and the norm does not assume its minimum on $M$ at $x$. Similarly, for sufficiently small negative $t,\|\alpha(t)\|>\|x\|$ and the norm does not assume its maximum on $M$ at $x$ either.

In the case where $B$ is a Hilbert space this condition yields a familiar result.

COROLlaRY 3. If $B$ is a Hilbert space, then $x$ is orthogonal to the tangent space to $M$ at $x$.

Proof. Since $\|x \mid A\|=\|x\|, x \in A$. 
3. We can represent $R$ as the quotient of the unit disk $D$ under the Fuchsian group $\Gamma$. Write the boundary of $D$ as the disjoint union of the closed set $\Lambda(\Gamma)$ of limits of fixed points of $\Gamma$ and the relatively open set $\Phi(\Gamma)$ of points of discontinuity of $\Gamma$. Then $R^{*}=D \cup \Phi(\Gamma) / \Gamma$ is a Riemann surface with boundary whose interior is $R$, and any such surface is (conformally equivalent to) an open subset of $R^{*}$. Thus the boundary of $R^{*}$ is as large as possible.

If $f: R \rightarrow S$ is a quasiconformal map, we may represent $S$ also as the quotient of the disk by another Fuchsian group $\Delta$, and the map $f$ is covered by a quasiconformal map $F$ of the unit disk to itself which has a continuous extension $F^{*}$ on the closed disk covering a continuous extension $f^{*}: R^{*} \rightarrow S^{*}$ of $f$.

THEOREM 3. Two maps $f, g: R \rightarrow S$ are homotopic modulo the boundary if and only if they can be covered by maps $F^{*}$ and $G^{*}$ of the disk to itself which agree on the boundary of the disk.

Proof. First suppose $f$ and $g$ are homotopic modulo the boundary, and let $h^{*}(t): R^{*} \rightarrow S^{*}$ be the homotopy with $h^{*}(0)=f^{*}, h^{*}(1)=g^{*}$ and $h^{*}$ constant on $R^{*}-R$. Then by the Covering Homotopy Theorem we can cover $h^{*}(t)$ with a homotopy $H^{*}(t): D \rightarrow D$ which is constant on $\Phi(\Gamma)$.

For each $\gamma \in \Gamma$ there exists a $\delta \in \Delta$ with $H^{*}(0) \gamma=\delta H^{*}(0)$. Fix $x$ in the interior of $D$ and consider the two curves in $D$ given by $H^{*}(t) \gamma(x)$ and $\delta H^{*}(t) x$. Since both are liftings of the same curve in $S$ and both have the same initial point, they must agree. Thus $H^{*}(t) \gamma=\delta H^{*}(t)$ for all $t$.

But then $H^{*}(t)$ must be constant on the fixed points of $\Gamma$, and hence on the whole boundary of the disk. Therefore $F^{*}=H^{*}(0)$ and $G^{*}=H^{*}(1)$ cover $f$ and $g$ and agree on the boundary of the disk.

Converseiy suppose $F^{*}$ and $G^{*}$ agree on the boundary of the disk. Define $H^{*}(t)(z)$ to be the point which divides the noneuclidean line segment between $F^{*}(z)$ and $G^{*}(z)$ in the ratio $t:(1-t)$. Then $H^{*}(t)$ covers a homotopy

$$
h^{*}(t): R^{*} \rightarrow S^{*}
$$

between $f^{*}$ and $g^{*}$ constant on $R^{*}-R$.

Let $f_{n}: R \rightarrow S$ be a sequence of maps homotopic to $f$ modulo the boundary, with $\left\|\beta\left(f_{n}\right)\right\| \leqq k<1$. Cover $f$ and $f_{n}$ with maps $F^{*}$ and $F_{n}^{*}$ of the closed disk to itself which all agree on the boundary, and with $\left\|\beta\left(F_{n}\right)\right\| \leqq k<1$. Some subsequence of the $F_{n}^{*}$ will converge uniformly to a quasiconformal map $G^{*}$ (see Ahlfors [1]) which agrees with $F^{*}$ on the boundary of the disk, and which covers a quasiconformal map $g: R \rightarrow S$ homotopic to $f$ modulo the boundary. Moreover $\|\beta(g)\| \leqq \lim$ inf $\left\|\beta\left(f_{n}\right)\right\|$ so we may choose the $f_{n}$ to make $g$ extremal.

4. In order to apply Theorem 2, I need the following result, which occurs (implicitly) in Bers [3]. 
THEOREM 4. Let $N$ be the set of all Beltrami differentials of quasiconformal maps of $R$ onto itself homotopic to the identity map modulo the boundary. Then $N$ is an analytic submanifold of $L^{\infty}\left(\bar{K} K^{-1}\right)$ in a neighborhood of zero whose tangent space at zero is the subspace orthogonal to $A(R)$.

For completeness I shall outline the proof. Let $D$ be the unit disk and $D^{\prime}$ its complement in the sphere. Define

$$
P_{n} \mu(z)=(n ! / 2 \pi i) \int_{D} \mu(\zeta)(\zeta-z)^{-n-1} d \zeta \wedge d \zeta
$$

For $\mu \in L^{\infty}(D), P_{n} \mu$ is analytic in $D^{\prime}$ with a zero at infinity of order at least $n+1$, and $d / d z P_{n} \mu=P_{n+1} \mu$ in $D^{\prime}$. Moreover for $\mu \in L^{p}(D)$, any $p>2, P_{0} \mu$ is Höldercontinuous in the entire sphere and has generalized derivatives $\partial / \partial \bar{z} P_{0} \mu=\mu$ and $\partial / \partial z P_{0} \mu=P_{1} \mu$. In $D, P_{1}$ is a singular integral and by the Calderón-Zygmund inequality it is a bounded linear operator of $L^{p}(D)$ into itself, whose norm, by the Riesz convexity theorem, approaches 1 as $p$ approaches 2 . We can then prove (see Ahlfors [2, p. 97]) that

$$
w(\mu)(z)=z+P_{0}\left(I-\mu P_{1}\right)^{-1} \mu(z)
$$

is a quasiconformal map of the sphere to itself with Beltrami differential $\mu$ on $D$ and analytic on $D^{\prime}$.

Remembering that $R=D / \Gamma$, let $L^{\infty}(D, \Gamma)=L^{\infty}\left(\bar{K} K^{-1}\right)$ be the Banach subspace of $\mu \in L^{\infty}(D)$ with $\mu=(\mu \circ \gamma) \arg ^{-2} \gamma^{\prime}$ for all $\gamma \in \Gamma$. Also let $B\left(D^{\prime}, \Gamma\right)$ be the Banach space of all analytic functions $\varphi$ in $D^{\prime}$ with a zero of order at least 4 at infinity, which satisfy $\varphi=(\varphi \circ \gamma)\left(\gamma^{\prime}\right)^{2}$ and whose norm $\sup (z \bar{z}-1)^{2}|\varphi(z)|$ is finite. This is just the norm of the quadratic differential $\varphi(z) d z^{2}$ in the Poincaré metric on $D^{\prime}$. Let $[f]$ denote the Schwarzian derivative of $f$. Since $\mu$ is invariant under $\Gamma$, for each $\gamma \in \Gamma$ the map $w(\mu) \circ \gamma \circ w(\mu)^{-1}$ is an analytic homeomorphism of the sphere and hence is itself a Möbius transformation $\delta$. Then $w(\mu) \circ \gamma=\delta \circ w(\mu)$, and

$$
([w(\mu)] \circ \gamma)\left(\gamma^{\prime}\right)^{2}=[w(\mu)] .
$$

Moreover by a theorem of Nehari [6] on schlicht mappings sup $(z \bar{z}-1)^{2}|[w(\mu)]| \leqq 6$. Hence $\Lambda(\mu)=[w(\mu)]$ belongs to $B\left(D^{\prime}, \Gamma\right)$ and $\|\Lambda(\mu)\| \leqq 6$.

Lemma 1. $\Lambda: L^{\infty}(D, \Gamma) \rightarrow B\left(D^{\prime}, \Gamma\right)$ is a complex analytic map and $D \Lambda(0)=P_{3}$.

Proof. Fix a point $z \in D$. Since $\left(I-\mu P_{1}\right)^{-1} \mu$ is a uniformly convergent power series in $\mu, w(\mu)(z), d / d z w(\mu)(z), \ldots, d^{3} / d z^{3} w(\mu)(z)$ are all analytic functions of $\mu$. Therefore so is $\Lambda(\mu)(z)$. Let $\gamma$ be the circle of radius 1 in the $t$-plane. By the Cauchy integral formula, for $\|\nu\|<1-\|\mu\|$,

$$
D \Lambda(\mu)(\nu)(z)=(1 / 2 \pi i) \int_{\gamma} \Lambda(\mu+t v)(z) / t^{2} d t
$$


Since $|\Lambda(\mu+t v)(z)| \leqq 6(z \bar{z}-1)^{-2}, D \Lambda(\mu)$ is a bounded complex linear map of $L^{\infty}(D, \Gamma)$ into $B\left(D^{\prime}, \Gamma\right)$. Also for $|c| \leqq 1 / 2$

$$
\begin{aligned}
\mid \Lambda(\mu+c \nu)(z)-\Lambda(\mu)(z)-D \Lambda & (\mu)(c \nu)(z) \mid \\
& \leqq(1 / 2 \pi) \int_{\nu}|\Lambda(\mu+t \nu)(z)|\left|(t-c)^{-1}-t^{-1}-c t^{-2}\right| d t \\
& \leqq 12 c^{2}(z \bar{z}-1)^{-2} .
\end{aligned}
$$

Therefore $\|\Lambda(\mu+c \nu)-\Lambda(\mu)-D \Lambda(\mu)(c \nu)\| \leqq 12 c^{2}$ so $\Lambda$ is in fact differentiable with derivative $D \Lambda(\mu)$. Since $D \Lambda(\mu)$ is complex-linear, $\Lambda$ is analytic. By evaluating at $z$ again we may compute $D w(0)(\mu)(z)=P_{0} \mu(z)$ and $D \Lambda(0)(\mu)(z)=P_{3} \mu(z)$. Therefore $D \Lambda(0)=P_{3}$.

Define a continuous linear map $S: B\left(D^{\prime}, \Gamma\right) \rightarrow L^{\infty}(D, \Gamma)$ by

$$
S \varphi(z)=c(1-z \bar{z})^{2} \bar{z}^{-4} \varphi\left(\bar{z}^{-1}\right)
$$

Using a reproducing formula for analytic functions (see Bers [3, Lecture 3, p. 6]), $D \Lambda(0) \circ S$ is the identity for an appropriate choice of the constant $c$. This proves that $D \Lambda(0)$ maps $L^{\infty}(D, \Gamma)$ onto $B\left(D^{\prime}, \Gamma\right)$ and its kernel is a closed split subspace. By the inverse function theorem $\Lambda^{-1}(0)$ is an analytic submanifold in a neighborhood of zero.

LEMMA 2. $\Lambda^{-1}(0)=N$.

Proof. First suppose $\mu \in \Lambda^{-1}(0)$. Then the Schwarzian derivative of $w(\mu)$ is zero on $D^{\prime}$, so $w(\mu)$ agrees on $D^{\prime}$ with a Möbius transformation $A$. Let $w=A^{-1} \circ w(\mu)$. Then $w$ is $\mu$-quasiconformal on $D$ and the identity on $D^{\prime}$. Since $\mu \in L^{\infty}(D, G)$, $w$ covers a $\mu$-quasiconformal map of $R$ onto itself homotopic to the identity modulo the boundary.

Conversely any such map can be lifted to a quasiconformal map $w$ of $D$ onto itself which leaves the boundary fixed. Let $\mu=\beta(w)$, and extend $w$ to be the identity in $D^{\prime}$. Then $w(\mu) w^{-1}$ is an analytic one-to-one map of the sphere onto itself and therefore is a Möbius transformation whose Schwarzian derivative in $D^{\prime}$ is $\Lambda(\mu)=0$.

Finally

$$
D \Lambda(0)(\mu)=P_{3} \mu=(6 / 2 \pi i) \int_{D} \mu(\zeta) /(\zeta-z)^{4} d \zeta \wedge d \zeta
$$

and the functions $(\zeta-z)^{-4}$ are dense in $A(D)$, the integrable analytic functions in $D$. Moreover we know (see Earle [4]) that each element of $A(R)$ is a Poincaré series of an element of $A(D)$. Therefore $T_{0} N=\operatorname{Ker} D \Lambda(0)$ is the subspace of $L^{\infty}\left(\bar{K} K^{-1}\right)$ orthogonal to $A(R)$. 
5. The composition of two quasiconformal maps is again quasiconformal. If $\mu=\beta(f)$ and $\nu=\beta(g)$ then

$$
\begin{aligned}
\partial(g \circ f) / \partial z & =\partial g / \partial w \partial f / \partial z+\partial g / \partial \bar{w} \partial \bar{f} / \partial z \\
& =\partial g / \partial w \partial f / \partial z\left(1+\bar{\mu} \nu \arg ^{-2} \partial f / \partial z\right) \\
\partial(g \circ f) / \partial \bar{z} & =\partial g / \partial w \partial f / \partial \bar{z}+\partial g / \partial \bar{w} \partial \bar{f} / \partial \bar{z} \\
& =\partial g / \partial w \partial f / \partial z\left(\mu+\nu \arg ^{-2} \partial f / \partial z\right) .
\end{aligned}
$$

Here $f^{\#} \nu=\nu \arg ^{-2} \partial f / \partial z$ is the pull-back of $\nu d \bar{w} / d w$ as a tensor. We then have

$$
\beta(g \circ f)=\left(\mu+f^{\#} \nu\right) /\left(1+\bar{\mu} f^{\#} \nu\right) .
$$

We may regard this as a calculation in local coordinates for Riemann surfaces. Let $R, S$, and $T$ be Riemann surfaces, $f: R \rightarrow S$ and $g: S \rightarrow T$ quasiconformal maps, and $K$ and $J$ the complex cotangent bundles on $R$ and $S$. The tensor pull-back defines a linear isometric isomorphism $f^{\#}: L^{\infty}\left(\bar{J} J^{-1}\right) \rightarrow L^{\infty}\left(\bar{K} K^{-1}\right)$. It is an isometry because $\left|f^{\#} \nu_{\alpha}\right|=\left|\nu_{\beta}\right| \circ f_{\beta \alpha}$, and an isomorphism because $\left(f^{\#}\right)^{-1}=\left(f^{-1}\right)^{\#}$. Then if $\mu=\beta(f)$ and $\nu=\beta(g)$ we have as before

$$
\beta(g \circ f)=\left(\mu+f^{\#} \nu\right) /\left(1+\bar{\mu} f^{\#} \nu\right) .
$$

This suggests that we define a map of the Beltrami differentials on $S$ to the Beltrami differentials on $R$ by

$$
C(f)(\nu)=\left(\mu+f^{\#} \nu\right) /\left(1+\bar{\mu} f^{\#} \nu\right) .
$$

The Beltrami differentials on $R$ are the points in the unit ball in $L^{\infty}\left(\bar{K} K^{-1}\right)$, which I denote by $B(R)$. The map $C(f): B(S) \rightarrow B(R)$ is analytic. Indeed $\left(1+\bar{\mu} f^{\#} \nu\right)^{-1}$ admits a uniformly convergent power series since $\left\|\bar{\mu} f_{\nu} \nu\right\|<1$. Moreover $\beta(g \circ f)=$ $C(f) \beta(g)$, and since every Beltrami differential is the Beltrami differential of some quasiconformal map, it follows that $C(g \circ f)=C(g) \circ C(f)$. Therefore, $C(f)^{-1}=$ $C\left(f^{-1}\right)$ and $C(f)$ is bi-analytic.

Let $d$ be the Poincaré metric in the disk $D$, given by

$$
d(z, w)=(1 / 2) \log (1+r) /(1-r) \quad \text { where } r=|(z-w) /(1-\bar{z} w)| .
$$

If $\nu$ and $\pi$ are two Beltrami differentials and $\alpha$ and $\beta$ are two coordinate charts then since

$$
v_{\beta}=\nu_{\alpha} \arg ^{2}\left(d z_{\beta} / d z_{\alpha}\right) \text { and } \pi_{\beta}=\pi_{\alpha} \arg ^{2}\left(d z_{\beta} / d z_{\alpha}\right)
$$

the number $d(\nu(x), \pi(x))=d\left(v_{\alpha}(x), \pi_{\alpha}(x)\right)$ is invariantly defined for almost all $x$. Define a metric on $B(R)$, the Beltrami differentials on $R$, by

$$
\tau(\nu, \pi)=\text { ess sup } d(\nu(x), \pi(x))
$$

where the essential supremum is taken over almost all $x$ in $R$. This metric is natural in the sense that it makes each map $C(f): B(S) \rightarrow B(R)$ an isometry (if we define a metric $\tau$ on $B(S)$ in the same way). To establish this result it is necessary only to observe that in terms of a local coordinate chart the map $C(f)$ is induced by a Möbius transformation from the unit disk in each fibre of $L^{\infty}\left(\bar{J} J^{-1}\right)$ to the unit 
disk in the corresponding fibre of $L^{\infty}\left(\bar{K} K^{-1}\right)$, and the Poincare metric is invariant under Möbius transformations. This metric is shown to be induced by a Finsler structure on $B(R)$ by Earle and Eells in [5].

We shall need the following estimate comparing the metric $\tau$ with the $L^{\infty}$ norm.

THEOREM 5. Let $\nu$ and $\pi$ be Beltrami differentials. Then

$$
\tau(\nu, \pi)-\tau(\nu, 0) \leqq\|\nu-\pi\|-\|\nu\|+o(\|\pi\|)
$$

where $o(t) / t \rightarrow 0$ as $t \rightarrow 0$.

Proof. The Poincaré metric is very close to the Euclidean metric at the origin, so that

$$
d(z, w)-d(z, 0) \leqq|z-w|-|z|+o(|w|)
$$

where $o(t) / t \rightarrow 0$ as $t \rightarrow 0$. To prove this, regard $d(z, w)$ and $|z-w|$ as two functions of $w$ for fixed $z \neq 0$ and evaluate the derivatives $\partial / \partial w$ at $w=0$. In both cases a laborious calculation yields $-\bar{z} / 2|z|$. Since $d(z, w)$ and $|z-w|$ are real the derivatives $\partial / \partial \bar{w}$ are obtained by conjugation. Hence both partial derivatives agree at $w=0$ and are continuous, so the estimate follows from the Mean Value Theorem. On the other hand, if $z=0$ we may regard

$$
d(0, w)=(1 / 2) \log (1+|w|) /(1-|w|)
$$

as a function of $|w|$, and taking its derivative at $|w|=0$ we again obtain the required estimate.

By replacing $o(t)$ by $\sup \{o(u) \mid 0 \leqq u \leqq t\}$ we may assume that the error estimate $o(t)$ is monotone nondecreasing. Then for the metric $\tau$ we have

$$
\begin{aligned}
\tau(\nu, \pi) & =\operatorname{ess} \sup d(\nu(x), \pi(x)) \\
& \leqq \operatorname{ess} \sup \{d(\nu(x), 0)+|\nu(x)-\pi(x)|-|\nu(x)|+o(|\pi(x)|)\} \\
& \leqq \text { ess sup }\{d(\nu(x), 0)-|\nu(x)|\}+\text { ess sup }|\nu(x)-\pi(x)|+\text { ess sup } o(|\pi(x)|) .
\end{aligned}
$$

But $d(z, 0)-|z|$ is a monotonic increasing function of $|z|$, since

$$
d / d r(1 / 2) \log (1+r) /(1-r)=1 /\left(1-r^{2}\right) .
$$

Therefore

$$
\tau(\nu, \pi) \leqq \tau(\nu, 0)-\|\nu\|+\|\nu-\pi\|+o(\|\pi\|)
$$

which proves the theorem.

6. Let $N$ be as before the set of all Beltrami differentials of quasiconformal maps of $R$ onto itself homotopic to the identity modulo the boundary.

TheOREM 6. Let $f: R \rightarrow S$ be extremal and $\mu=\beta(f)$. Then $\tau(\mu, 0) \leqq \tau(\mu, \pi)$ for all $\pi \in N$.

Proof. Suppose $\pi \in N$ and $\tau(\mu, \pi)<\tau(\mu, 0)$. We know that $\pi=\beta(g)$ for some quasiconformal map $g: R \rightarrow R$ homotopic to the identity modulo the boundary. 
If $H: R \times[0,1] \rightarrow R$ is a homotopy between $g$ and the identity fixing the boundary then $f \circ g^{-1} \circ H$ is a homotopy between $f$ and the map $k=f \circ g^{-1}$ which leaves the boundary fixed. Since $k \circ g=f, C(g) \beta(k)=\beta(f)$. Let $\mu=\beta(f)$ and $\lambda=\beta(k)$. Since $C(g)$ is an isometry and $C(g) 0=\beta(g)=\pi$,

$$
\tau(\lambda, 0)=\tau(C(g) \lambda, C(g) 0)=\tau(\mu, \pi)<\tau(\mu, 0) .
$$

But $\tau(\mu, 0)$ is a monotone increasing function of $\|\mu\|$ since $d(z, 0)$ is a monotone increasing function of $z$. Therefore $\|\lambda\|<\|\mu\|$. Since $\lambda=\beta(k)$ and $k$ is homotopic to $f$ modulo the boundary, $f$ is not extremal.

It is now easy to complete the proof of Theorem 1 by imitating the proof of Theorem 2, using the estimate in Theorem 5. Suppose that $f: R \rightarrow S$ is quasiconformal with Beltrami differential $\mu=\beta(f)$ but that $\|\mu \mid A(R)\|<\|\mu\|$. By the Hahn-Banach Theorem we can find $\nu \in L^{\infty}\left(\bar{K} K^{-1}\right)$ with $\nu|A(R)=\mu| A(R)$ and $\|\nu\|=\|\mu \mid A(R)\|<\|\mu\|$. Then $\mu-\nu \in A(R)^{\perp}$ and $A(R)^{\perp}$ is the tangent space at 0 to the analytic submanifold $N$. Consequently we can find a $C^{1}$ path $\alpha:(-\varepsilon, \varepsilon) \rightarrow N$ with $\alpha(0)=0$ and $D \alpha(0)(1)=\mu-\nu$. Then, restricting our attention to positive $t$,

$$
\|\alpha(t)-\alpha(0)-D \alpha(0)(t)\| / t=\|\alpha(t)-t \mu+t v\| / t \rightarrow 0 \text { as } t \rightarrow 0 .
$$

This makes $\|\alpha(t)\| \leqq K t$ for some constant $K$ and all sufficiently small $t$, so $o(\|\alpha(t)\|) / t \rightarrow 0$ as $t \rightarrow 0$ as well.

Now by the estimate of Theorem 5

$$
\tau(\mu, \alpha(t))-\tau(\mu, 0) \leqq\|\mu-\alpha(t)\|-\|\mu\|+o(\|\alpha(t)\|) .
$$

Also $\|\mu-\alpha(t)\| \leqq(1-t)\|\mu\|+t\|\nu\|+\|\alpha(t)-t \mu+t \nu\|$. Combining these inequalities with the estimates above we see that

$$
\tau(\mu, \alpha(t))-\tau(\mu, 0) \leqq \beta(t)-t(\|\mu\|-\|\nu\|)
$$

where $\beta(t) / t \rightarrow 0$ as $t \rightarrow 0$. Since $\|\mu\|-\|\nu\|>0$ we must have $\tau(\mu, \alpha(t))<\tau(\mu, 0)$ for all sufficiently small pcsitive $t$. Then since $\alpha(t) \in N$ it follows from Theorem 6 that the map $f: R \rightarrow S$ is not extremal.

\section{REFERENCES}

1. L. V. Ahlfors, On quasiconformal mappings, J. Analyse Math. 3 (1953/1954), 1-58.

2. —_, Lectures on quasiconformal mappings, Van Nostrand, New York, 1966.

3. L. Bers, On moduli of Riemann surfaces, Lecture Notes, Eidgenössische Technische Hochschule, Zürich, 1964.

4. C. Earle, A reproducing formula for integrable automorphic forms, Amer. J. Math. 88 (1966), 867-870.

5. C. Earle and J. Eells, Jr., On the differential geometry of Teichmüller spaces, J. Analyse Math. 19 (1967), 35-52.

6. Z. Nehari, The Schwarzian derivative and schlict functions, Bull. Amer. Math. Soc. 55 (1949), 545-551.

7. K. Strebel, Zur Frage der Eindeutigkeit extremaler quasiconformer Abbildungen des Einheitskreises, Comment. Math. Helv. 36 (1962), 306-323.

CORNELl UNIVERSity, ITHACA, NeW YoRK 